\title{
UTILIZACIÓN DE SUPLEMENTOS NUTRICIONALES PARA EL MANEJO DEL SOBREPESO Y OBESIDAD: UNA REVISIÓN DE LA EVIDENCIA
}

\author{
USE OF DIETARY SUPPLEMENTS FOR THE \\ MANAGEMENT OF OVERWEIGHT AND OBESITY: \\ A REVIEW OF THE EVIDENCE
}

\author{
Alejandra Wiedeman M., Erika Ochoa O., Eduard Maury S., Martin Gotteland \\ Laboratorio de Microbiología y Probióticos, \\ Instituto de Nutrición y Tecnología de los Alimentos (INTA), Universidad de Chile, Santiago, Chile.
}

\begin{abstract}
Simultaneously to the rapid increase of overweight and obesity a great number of products oriented to weight loss have emerged in the market. The aim of this study was to evaluate the scientific evidence supporting the use of three products designed for weight loss available in Chile: a lipidic emulsion that stimulates ileal brake, a protease inhibitor and an amylase inhibitor. The results indicate that the amylase and protease inhibitors efficiently inhibit the respective enzymatic activities in vitro; however, they do not affect weight control in clinical trials carried out in normal weight as well as in obese subjects. Thus, there is not enough evidence supporting the use of these products for weight control. On the other hand, the results concerning the lipidic emulsion are conflicting and more studies are needed to confirm its possible effect on weight control.
\end{abstract}

Key words: dietary supplement, overweight, obesity.

Este trabajo fue recibido el 13 de Diciembre de 2010 y aceptado para ser publicado el 19 de Mayo de 2011.

\section{INTRODUCCIÓN}

La población mundial enfrenta una epidemia de sobrepeso y obesidad y sus complicaciones metabólicas como la diabetes tipo 2 y las enfermedades cardiovasculares. Si bien anteriormente estas patologías afectaban principalmente las poblaciones de los países industrializados, la transición demográfica, epidemiológica y nutricional que ha ocurrido en las últimas décadas ha provocado su generalización a los países en desarrollo, donde frecuentemente coexisten con la malnutrición. Este fenómeno se debe principalmente al mejoramiento de la calidad vida (mejores sueldos, calefacción central, uso de automóviles, mayor disponibilidad de alimentos, entre otros), junto con la occidentalización de la dieta y la disminución de la actividad física (1).

El año 2005, la Organización Mundial de la Salud (OMS) estimaba que el sobrepeso afectaba a 1.6 billones y la obesidad a 400 millones de adultos en el mundo.
Asimismo, en sus proyecciones para el 2015 este organismo estima que el sobrepeso incremente hasta 2.3 billones de personas y que la obesidad casi se duplicaría (hasta 700 millones) (2). La población chilena no es la excepción; a consecuencia de la occidentalización de la dieta, el consumo de grasa ha ido aumentando con rapidez (1), mientras disminuye el consumo de granos enteros, legumbres, frutas y verduras. Tomando en cuenta que, además, el $90 \%$ de la población es sedentaria, la prevalencia de sobrepeso y obesidad en nuestro país ha alcanzado cifras de hasta $61.3 \%$ (38\% sobrepeso, $22 \%$ obesidad y $1.3 \%$ obesidad mórbida) (3).

En estas condiciones se hace difícil restablecer el equilibrio ponderal una vez que el individuo esta en sobrepeso u obeso. Generalmente, se requiere un equipo multidisciplinario que integre médicos, psicólogos, nutricionistas y monitores de educación física. El papel de las empresas de alimentos es también importante, pues 
deben desarrollar alimentos más saludables y equilibrados nutricionalmente, que se adecúen a la situación de salud pública que vive el país, sin dejar de ser atractivos para el consumidor.

Paralelamente al rápido aumento de la obesidad en Chile han surgido un sinnúmero de productos o suplementos dietarios que prometen, con mucha publicidad, perder peso o mantenerlo sin sacrificar el estilo de vida, es decir, manteniendo una dieta hipercalórica de calidad inadecuada y sin abandonar el sedentarismo. Las razones por las cuales un individuo decide iniciar el consumo de dichos suplementos para tratar de controlar su peso se relacionan con el estigma social de la obesidad, los beneficios que conlleva bajar de peso, la promesa de una solución rápida y poco demandante, así como la posibilidad de acceder a estos productos sin necesidad de consultar o ser supervisado por un profesional de la salud (4). A pesar de que no hay cifras disponibles en cuanto al consumo de estos productos en Chile, su multiplicación hace pensar que se trata de un negocio altamente rentable. Dichos productos tienen en general precios bastante elevados por lo cual su consumo regular representa una carga para el presupuesto individual o familiar, a expensa de la compra y consumo de alimentos con mejor calidad nutricional.

Es importante, por lo tanto, evaluar en forma crítica estos suplementos, indagando las bases científicas que justifiquen su uso para el control ponderal, en particular los mecanismos de acción capaces de explicar sus efectos y los resultados que comprueban su real eficiencia. En base a estas premisas, el objetivo de este trabajo es evaluar la evidencia científica que apoya el uso de tres suplementos dietarios comercializados en Chile y que son destinados al control de peso a través de la regulación del apetito/saciedad (una emulsión lipídica y un inhibidor de proteasas) o de la disminución de la digestión de hidratos de carbono (un inhibidor de amilasa).

\section{METODOLOGÍA}

Se realizó una búsqueda sistemática y actualizada (hasta octubre de 2010) en la base de datos PUBMED (http://www.ncbi.nlm.nih.gov/sites/entrez) usando las siguientes palabras clave: fabuless, olibra, ileal brake, slendesta, protease inhibitors, cholecystokinin, phaseolamina, phaseolus vulgaris, phase 2, inhibitor of $\alpha$-amylase, inhibitor absorption starch, starch blocker, satiety, eating, weight loss, weight control, obesity.

Cabe destacar que por el escaso número de estudios que se han efectuado ( 8 olibra, 3 en inhibidores de proteasas y 13 en inhibidores de amilasa) no permite hacer análisis estadístico.

\section{RESULTADOS \\ 1. Olibra: una emulsión lipídica para la estimulación del freno ileal a-Base fisiológica de la utilización del producto}

Olibra es una emulsión patentada que contiene $42 \%$ de grasa en forma de aceite de palma recubierta por galactolípidos provenientes de aceite de avena. Esta emulsión ha sido incorporada a alimentos como yogurts o leche ("concepto Fabuless") o representa la base de productos como Slim Kiup que llegó a Chile en 2008. Dicho producto se vende en formato individual de 7.5 $\mathrm{ml}$, y se recomienda el consumo de dos dosis por día en los horarios de desayuno y almuerzo; propone reducir la ingesta calórica en un $30 \%$, aumentando la saciedad a través del freno ileal (5), refiriéndose a la capacidad que tienen ciertos nutrientes de estimular la secreción de hormonas digestivas tales como el GIP, el GLP-1 y el PYY por células enteroendocrinas del epitelio ileal. El GIP inhibe el vaciamiento gástrico, aumentando la sensación de plenitud gástrica y disminuyendo la ingesta de más alimentos mientras que el PYY y el GLP-1 ejercen efectos anorexigénicos a nivel del sistema nervioso central, aumentando la saciedad (6) es decir posponiendo el consumo de la siguiente comida.

La digestión de los triglicéridos dietarios es un proceso que comienza en el estómago con la lipasa gástrica y continúa en el duodeno con la lipasa pancreática (7); libera ácidos grasos que luego son absorbidos por los enterocitos. Se trata de un proceso eficiente que ocurre principalmente en el intestino proximal. Se estima que en el caso del Olibra, los galactolípidos que recubren la emulsión lipídica son más resistentes a la digestión enzimática por lo cual la liberación del aceite de palma y la posterior digestión y absorción de sus triglicéridos constitutivos ocurre más tardíamente en los segmentos distales del tubo digestivo. Un estudio reciente en voluntarios sanos que recibían una perfusión intragástrica de Olibra observó, a partir de muestras de contenido intraluminal recolectadas a nivel yeyunal, que esta emulsión genera la formación de cristales de ácidos grasos saturados, los cuales postergan la absorción de los ácidos grasos hacia los segmentos más distales del intestino (8). Dichos ácidos grasos son potentes agentes estimuladores de las células enteroendocrinas productoras de GLP-1, PYY y GIP, mecanismo por el cual favorecerían la saciedad.

\section{b- Estudios y ensayos clínicos con Olibra}

Varios ensayos clínicos han estudiado el efecto del consumo de yogurt con Olibra sobre la saciedad (tabla 1), comparado su efecto con el de un yogurt placebo con el mismo contenido de energía y grasa digestible. En estos 


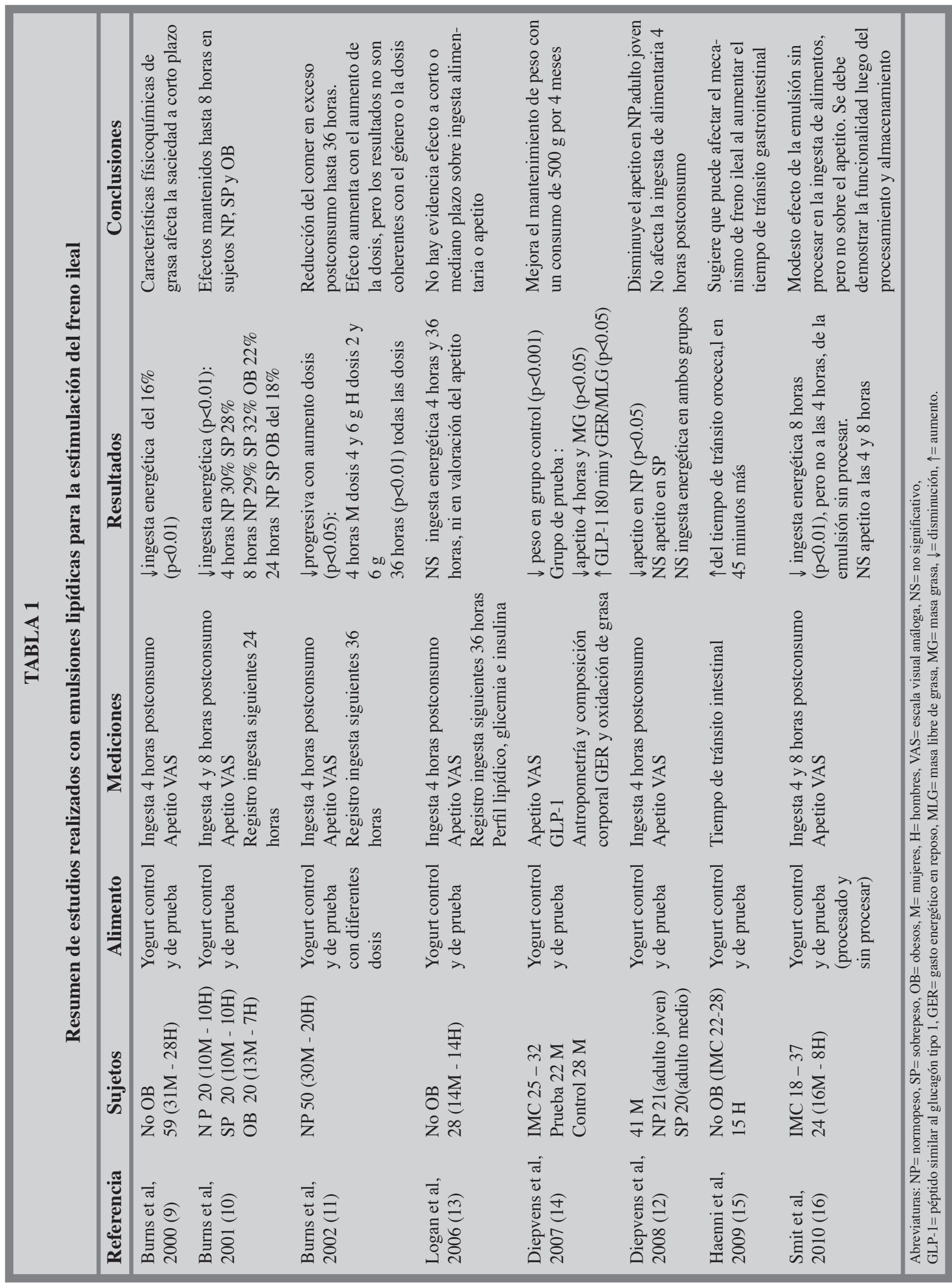


estudios la intensidad de la sensación de apetito/saciedad fue determinada a través de escala visual subjetiva. En estos ensayos, la intensidad de la saciedad se determinó a corto (9-12), mediano (13) y largo plazo (14), definidos por tiempos de 4 horas, 3 semanas y 4 meses, respectivamente. Tres de estos estudios sugieren que el consumo de Olibra ( $5 \mathrm{~g}$ en $200 \mathrm{~g}$ de yogurt) afecta la saciedad a corto plazo, disminuyendo significativamente el consumo de energía y de macronutrientes incluyendo a la grasa, tanto en individuos normopeso como en aquellos con sobrepeso y obesos (9-11). La administración de un yogurt con distintas cantidades de Olibra $(2,4$ y $6 \mathrm{~g})$ a voluntarios normopeso disminuyó el consumo de energía en un 21 , 25 y $30 \%$, respectivamente, en las siguientes 24 horas, comparado con el yogurt placebo sin la emulsión (11). Dicho efecto fue significativo con la dosis mínima de 2 $\mathrm{g}$ pero no se observaron diferencias significativas entre las distintas dosis utilizadas; es interesante destacar que la inducción de la saciedad por el Olibra fue mayor en las mujeres que en los varones para una misma dosis. En oposición con estos resultados, Logan et al. no observaron efectos del Olibra a corto y mediano plazo sobre el apetito y el consumo de alimentos en sujetos normopeso (13). Diepvens et al. observaron que el consumo de Olibra ( $10 \mathrm{~g}$ en $250 \mathrm{~g}$ de yogurt) disminuye el apetito a corto plazo en mujeres normopeso pero no en obesas, pero que no reducía la ingesta energética en estos dos grupos; tampoco observaron efectos a mediano plazo (12). En otro estudio, los mismos autores evaluaron por 18 semanas y en 50 mujeres con sobrepeso los efectos del consumo de yogurt con Olibra sobre la mantención del peso corporal, posterior a un período de 6 semanas de pérdida de peso (14). Observaron que los sujetos con Olibra mantuvieron su peso corporal mientras que los sujetos controles lo aumentaron significativamente; además los sujetos tratados tuvieron una menor sensación de hambre y mayores concentraciones circulantes de GLP-1 después del consumo de yogurt con Olibra y su masa grasa disminuyó significativamente en comparación al grupo control. Por otra parte, dado que una de las consecuencias de la estimulación del "freno ileal" es enlentecer el tránsito gastrointestinal, Haeni et al midieron el tiempo de tránsito oro-cecal de sujetos sanos después del consumo de yogurt con y sin Olibra (15), observando que éste efectivamente se alargaba en 45 minutos después del consumo de la emulsión. Finalmente, para aclarar si el procesamiento (pasteurización y homogenización) del alimento que sirve de vehículo para el Olibra puede afectar su estructura y explicar los resultados contradictorios obtenidos en los ensayos clínicos anteriormente descritos, se realizó un ensayo clínico en 24 voluntarios sanos utilizando Olibra procesado o sin procesar (16).
Se observó un efecto moderado sobre el consumo de alimento sólo con Olibra sin procesar, sin embargo sin efecto sobre el apetito. Estos resultados muestran la importancia que pueden tener los procesamientos aplicados al alimento en determinar la estabilidad de Olibra y sus efectos sobre el control ponderal.

\section{c- Conclusión sobre el uso de Olibra}

Los ensayos clínicos que evaluan el efecto de Olibra publicados en la literatura muestran resultados contradictorios; por lo tanto no parece haber suficiente evidencia en la actualidad para avalar el efecto de esta emulsión sobre el control del apetito/saciedad, el consumo de alimentos y el control ponderal. Ciertos procesos tecnológicos, que parecen afectar la estabilidad de la emulsión en el alimento, podrían explicar estas discrepancias.

\section{Inhibidores de Proteasas para el control del apetito a- Base fisiológica de la utilización de los inhibidores de proteasas}

Los inhibidores de proteasas comercializados bajo el nombre de Slendesta son elaborados por la empresa norteamericana Kemin, dedicada a la elaboración de ingredientes para la industria agro-alimentaria. Slendesta esta comercializado en Chile como Benicia bajo forma de comprimidos o de polvo para ser reconstituido con leche descremada. Estas presentaciones aportan 600 $\mathrm{mg}$ de Slendesta (es decir $30 \mathrm{mg}$ de PI2, el ingrediente activo). Se recomienda tomar 2 comprimidos 1 hora antes de cada comida principal (4 cápsulas por día), o consumir la bebida reconstituida entre las comidas como colación (1 a 2 veces al día), por un mínimo de tres meses. Slendesta se describe como un extracto estandarizado de papa cuyo compuesto activo denominado PSPI o PI2 o POT II (un inhibidor de serina proteasa), permitiría mantener elevados los niveles plasmáticos de colescistokinina (CCK).

Dicha hormona es liberada por células enteroendocrinas del duodeno y del yeyuno en respuesta a la presencia intraluminal de ácidos grasos y de péptidos dietarios. La CCK está involucrada en la estimulación de la secreción bilio-pancreática, en la regulación de la función gástrica (vaciamiento gástrico y secreción de $\mathrm{HCl}$ ), en los procesos de termorregulación y también tiene efectos anorexigénicos $(17,18)$. Las enzimas pancreáticas tripsina y quimotripsina, cuya secreción es estimulada por la CCK, ejercen una retroinhibición sobre la liberación de esta hormona, regulando su concentración circulante y por ende sus efectos. El inhibidor de proteasas del Slendesta, PI2, bloquearía dichas enzimas, fijándose a ellas e impidiendo que ejerzan su control 
negativo sobre la liberación de CCK; este fenómeno permitiría mantener elevada la concentración circulante de CCK, estimulando la saciedad temprana, disminuyendo la ingesta de alimentos y de energía y, por ende, favoreciendo el control ponderal.

En el marco de esta problemática, es importante recordar que el apetito y la saciedad son controlados a través de interacciones complejas que integran señales periféricas con el sistema nervioso central a través de sus sistemas neuropeptidérgicos, monoaminérgicos y endocanabinoides (19). Varias hormonas producidas, entre otros, por el tracto gastrointestinal y por el tejido adiposo, tales como la CCK, el GLP-1, el PYY, la grelina y la leptina son capaces de regular el apetito/saciedad y por lo tanto la ingesta de alimentos (20). La complejidad de este sistema favorece la redundancia de tal forma que el bloqueo de una de estas señales no necesariamente promueve la saciedad debido a la existencia de numerosos mecanismos de compensación. De no ser así, la obesidad monogénica sería más común.

\section{b- Estudios y ensayos clínicos con los inhibidores de proteasas}

Si bien existen muchos estudios acerca del rol de PI2 en la papa, los estudios relativos a sus efectos fisiológicos en animales y seres humanos son escasos. La inocuidad de PI2 ha sido evaluada en modelos animales; no se han observado efectos tóxicos después del consumo agudo del producto pero algunos estudios sugieren que su consumo a largo plazo podría inducir hipertrofia y neoplasia pancreática como consecuencia del efecto trófico de la CCK sobre este órgano (21); no existen datos disponibles en lo que se refiere al ser humano. Recientemente Komarnytsky et al (22) mostraron que la administración aguda a ratas normopeso de $100 \mathrm{mg} /$ $\mathrm{kg}$ /día de PI2 reducía el consumo de alimentos en las 2 horas post-tratamiento, atrasaba el vaciamiento gástrico y disminuía la actividad proteolítica en el duodeno. La administración regular del inhibidor resultó en la disminución del peso de los animales y en el aumento de sus niveles de CCK. En la línea celular STC-1, el inhibidor no afectó la expresión génica de CCK ni su liberación. El consumo de dosis crecientes del inhibidor (33-266 $\mathrm{mg} / \mathrm{kg} /$ día) no mostró efectos adversos, confirmando los estudios anteriores de toxicidad a corto plazo.

A pesar de que la información entregada por la empresa acerca del Slendesta habla de numerosos ensayos clínicos sobre más de 500 sujetos, la búsqueda en el sistema de bibliografía informatizada utilizada en este artículo muestra sólo la existencia de tres ensayos clínicos publicados en la literatura científica, dos de ellos hace ya más de 14 años. En el primer estudio, 11 sujetos normopeso recibieron en días distintos y en forma aleatoria 3 tratamientos: 1) una sopa con un alto contenido de proteína (estimulante de la liberación de CCK) y con $1.5 \mathrm{~g}$ de PI2, 2) la misma sopa sin inhibidor de proteasa, y 3) nada. Cinco minutos después de cada tratamiento se les ofreció un almuerzo ad libitum que incluía sus alimentos preferidos. Los niveles de CCK no fueron determinados en este estudio. Los autores reportan una disminución significativa del $17.5 \%$ en la ingesta calórica de los sujetos después del consumo de la sopa con PI2, mayor a la observada con la sopa sola y con el control (23); sin embargo el análisis del aporte calórico total del día no mostró diferencias entre los tres tratamientos. La administración de PI2 tampoco disminuyó la sensación de apetito, evaluada mediante uso de escala visual, comparado con los controles. De estos resultados no se puede inferir si el menor consumo calórico inducido por PI2 observado a corto plazo podría contribuir al control ponderal de los sujetos a largo plazo. El segundo estudio realizado en 6 sujetos diabéticos muestra que PI2 (1.5 g) atrasa el vaciamiento gástrico, disminuye los niveles post-prandial de glucosa, insulina y GIP y aumenta los niveles de CCK en estos pacientes (24); sin embargo, los autores no evaluaron la ingesta calórica de los sujetos ni la sensación de apetito/saciedad en este estudio lo que limita su impacto. Debido al número reducido de sujetos reclutados (17 para los dos estudios), estos dos estudios solo pueden ser considerados como estudios pilotos. El hecho que ambos estudios hayan sido realizados para evaluar efectos agudos y no efectos a mediano/largo plazo del consumo de PI2 es limitante para determinar el real impacto de este producto, tomando en cuenta la capacidad de adaptación del organismo y la redundancia y multiplicidad de los mecanismos fisiológicos implicados en la regulación del apetito/saciedad.

Finalmente, Peters et al. realizaron recientemente un ensayo clínico controlado por placebo y en crossover en 24 individuos que ingirieron una "minibebida" con 30mg de PI2 o un placebo 120 minutos (estudio 1) ó 30 minutos (estudio 2) antes de un almuerzo ad libitum o 30 minutos antes de un almuerzo fijo (estudio 3) (25). Los sujetos reportaron la intensidad de la sensación de saciedad (estudios 1 y 3 ) y su consumo de alimentos (estudios 1 y 2); las concentraciones de CCK y de glucosa fueron determinadas. A pesar de que los autores observaron que la minibebida con PI2 ejercía efectivamente una actividad inhibidora de proteasa en condiciones in vitro imitando las condiciones del intestino, mostraron que dicho producto no afectaba los parámetros estudiados en humanos, comparado con el placebo. En cuanto a los demás estudios citados por la empresa Kemin, se trata de 9 ensayos clínicos realizados en más de 500 sujetos 
sanos y/o obesos, todos realizados por los mismos autores entre 2004 y 2005. Los resultados obtenidos son presentados por la empresa bajo la misma forma que un artículo publicado en una revista científica pero sin serlo. De estos 9 ensayos, 7 han sido desarrollados en forma aleatorizada, en doble ciego y controlados por placebo y 2 son estudios abiertos. Se evaluaron los efectos del consumo del producto sobre el peso, la circunferencia de cadera y de cintura en 6 estudios (incluyendo los 2 abiertos), sobre la saciedad en 1 (a través de una evaluación subjetiva), sobre la glicemia e insulinemia en 1 y sobre los niveles de CCK en 1. En ninguno de los 6 estudios que evaluaron el efecto del producto sobre el peso se controló el consumo de alimento de los sujetos. En los 2 estudios abiertos (que por lo tanto tienen poco valor científico) se observan los mayores efectos del PI2 sobre el peso (pérdida de 3 y $5.3 \mathrm{~kg}$ ). La variabilidad de los datos obtenidos es alta y el análisis estadístico de los resultados carece de rigurosidad y tiende a sobrevalorar los efectos observados. Algunos de los resultados son incoherentes (incremento de la saciedad con una dosis de $15 \mathrm{mg}$ de PI2 y no con $30 \mathrm{mg}$, pero pérdida de peso corporal con $30 \mathrm{mg}$ y no con $15 \mathrm{mg}$ ) sin más explicación.

\section{c- Conclusión sobre el uso de los inhibidores de proteasas}

La evidencia científica que avala el uso de Slendesta para el control ponderal es claramente insuficiente. Se han publicados 3 ensayos clínicos, 2 que muestran un efecto del producto sobre la saciedad en un total de 17 sujetos pero no evaluaron su efecto sobre el control de peso, y uno cuyos resultados fueron negativos. El resto de los ensayos clínicos citados por la empresa no han sido publicados en revistas científicas, lo cual hace dudar de su calidad, ya que a través del proceso de publicación los estudios son evaluados y seleccionado por pares científicos en función de la calidad de su diseño, metodología, de los resultados obtenidos y de su interpretación.

\section{Inhibidores de amilasas \\ a- Base fisiológica de la utilización de los inhibidores de amilasa}

Se estima que en los países occidentales se consume entre 200 y $400 \mathrm{~g}$ de carbohidratos por día, principalmente como almidón (60\%); su sobreconsumo se ha asociado al sobrepeso y obesidad (26). Entre el 70 y el $80 \%$ del almidón es constituido por amilopectina, una cadena lineal larga de monómeros de glucosa unidos por enlaces a-1,4 y con ramificaciones en a- 1,6 cada 20-30 residuos de glucosa. La amilopectina es digerida en forma eficiente por la amilasa pancreática en el duodeno y yeyuno proximal, liberando glucosa que es rápidamente absorbida (27). El 20-30\% restante del almidón lo constituye la amilosa, una cadena lineal de glucosa unidas por enlaces a-1,4 que forma una estructura tridimensional en hélice cuyas espiras son estabilizadas por puentes hidrógeno. En consecuencia, el proceso de hidrólisis de la amilosa y de liberación de glucosa por acción de la amilasa es más lento que en el caso de la amilopectina y ocurre a lo largo del intestino delgado próximal. Por esta razón, el índice glicémico de la amilosa es más bajo que el de la amilopectina, siendo la amilosa considerada como la fracción del almidón "resistente" a la digestión (27). Es importante destacar que el tipo de preparación y cocción del alimento que contiene almidón influye sobre la digestibilidad y contenido de almidón resistente; en individuos sanos se estima que alrededor del $20 \%$ del almidón consumido llega al colón (28). El uso de inhibidores de amilasa se basa en el hecho que el almidón no digerido por la amilasa se pierde en las deposiciones con las calorías correspondientes, lo cual debería, por lo tanto, resultar a largo plazo en una disminución del peso para el consumidor.

\section{b- Estudios y ensayos clínicos con inhibidores de amilasa}

En los años 70 se empezaron a estudiar numerosos inhibidores de amilasas principalmente provenientes de cereales (trigo y centeno, maíz) y legumbres (phaseolus vulgaris) $(29,30)$. No obstante, sólo a partir de la siguiente década aparecieron en el mercado los "bloqueadores de almidón" prometiendo a los consumidores una reducción del peso y un mejoramiento en su perfil metabólico (regulación de la glicemia e insulinemia). Los extractos purificados del poroto común son los más utilizados en los productos comercializados en forma de tabletas o cápsulas (Phase2 ${ }^{\mathrm{TM}}$ y Phaseolamin $2250^{\mathrm{TM}}$ ). Las dosis diarias recomendadas para estos productos corresponden a 2 cápsulas 15 minutos antes de las comidas, con un máximo de 6 al día . El compuesto activo, la phaseolamina, es una glicoproteína de alrededor de 45.000 daltons que inhibe en forma no competitiva la amilasa, disminuyendo la digestión del almidón en estudios in vitro e in vivo $(31,32)$. Sin embargo, el efecto real de los inhibidores de amilasa comerciales ha sido muy controversial, ya que diversos estudios realizados en los años 80 han reportado resultados incongruentes y poco concluyentes. Hollenbeck et al. realizaron un estudio en 8 sujetos alimentados con una dieta con $58 \%$ de carbohidratos (50\% almidón, $50 \%$ sacarosa) en presencia de un inhibidor de amilasa o de un placebo (32). No observaron diferencias significativas entre ambos tratamientos en los resultados del test de hidrógeno en aire espirado (Hydrogen Breath Test, HBT) que mide la 
malabsorción de almidón a nivel intestinal y su llegada al colon, ni en la sintomatología digestiva (borborigmos, distensión intestinal, entre otros) ni tampoco en los cambios de glicemia y respuesta insulínica, indicando que la phaseolamina fue ineficiente en reducir o retardar la digestión de los $50 \mathrm{~g}$ de almidón consumidos.

Resultados similares fueron descritos por Layer et al (33) en 4 voluntarios en quienes se colocó una sonda oroileal y quienes debieron ingerir $50 \mathrm{~g}$ de almidón de arroz con placebo al día 1 y la misma cantidad de almidón con un inhibidor de amilasa al día 2. Con la administración del inhibidor se observó una reducción de $95 \%$ de la actividad amilasa en las muestras recolectadas desde el duodeno, yeyuno e íleon durante las primeras dos horas; no obstante, la actividad enzimática se restableció a niveles similares al control en el siguiente período de dos horas. Dichos resultados concuerdan con los resultados del HBT que mostró un incremento considerable de los niveles de hidrógeno espirado durante las primeras dos horas del estudio.

En otro estudio se administraron distintas dosis del inhibidor por una semana; la excreción de hidrógeno fue mayor con el inhibidor en comparación con el placebo (34); sin embargo, la ausencia de almidón no-digerido en las deposiciones de estos sujetos indica que este fue probablemente completamente fermentado por la microbiota colónica y que la energía correspondiente fue rescatada por el organismo en forma de ácidos grasos volátiles. Además, la excreción de hidrógeno disminuyó en un $60 \%$ después de una semana, sugiriendo un posible aumento de la secreción de amilasa pancreática para compensar la fracción inhibida por la phaeseolamina. Tanto en el estudio de Layer como en el de Boivin se observaron una disminución significativa de la glicemia postprandial. Las concentraciones plasmáticas de polipéptido inhibidor gástrico (GIP), péptido $\mathrm{C}$ e insulina fueron también significativamente menores con el consumo del inhibidor de $\alpha$-amilasa. Resultados similares fueron descritos por Vinson et al.: una dosis de $1.5 \mathrm{~g}$ de phaseolamina comercial (Phase $2^{\mathrm{TM}}$ ) redujo la glicemia post-prandial en un 66\% (35). Bo Linn et al. describieron similares hallazgos, reportando que la excreción fecal de energía como carbohidratos no variaba con el inhibidor comparado con el control (36). A diferencia de estos resultados, Boivin et al observaron un incremento significativo en las pérdidas fecales de carbohidratos durante el consumo de inhibidores comparado con el periodo control, pero que, sin embargo, resultó ser insignificante respecto a la cantidad de carbohidratos ingeridos en la dieta (37).

Por otro lado, Díaz et al. determinaron el efecto de un inhibidor de amilasa $(1 \mathrm{~g} / \mathrm{d})$ sobre el peso corporal de mujeres obesas chilenas que recibían una dieta controlada por 5 meses (38). Las variaciones del peso fueron similares en ambos grupos, tratado y placebo, de acuerdo a lo esperado considerando la dieta hipocalórica prescrita. Además, los autores no encontraron diferencias en los resultados del HBT entre ambos tratamientos y tampoco observaron un efecto significativo del inhibidor sobre la reducción de la glicemia. Thom et al. observaron que la administración de un inhibidor de $\alpha$-amilasa a voluntarios disminuya el peso corporal, índice de masa corporal y porcentaje de grasa corporal comparado con el grupo placebo (39). No obstante, cabe destacar que el producto administrado también contenía Garcinia Cambogia, una sustancia capaz de reducir la absorción de la grasa en el intestino delgado, lo que podría en parte explicar dichos resultados. Finalmente, Udani et al. realizaron un ensayo clínico en 39 sujetos obesos que ingirieron $1.5 \mathrm{~g}$ de inhibidor de amilasa 2 veces al día con las comidas por 8 semanas, sin observarse cambios significativos en el peso corporal de los sujetos (43).

\section{c- Conclusión sobre el uso de los inhibidores de amilasa}

La evidencia científica descrita anteriormente no avala el uso de los inhibidores de amilasa para el control ponderal. Ninguno de los ensayos clínicos realizados muestra una pérdida de peso en los sujetos que consumen dicho producto. Si bien se detecta (mediante el HBT) un cierto grado de malabsorción del almidón con el inhibidor de amilasa, prácticamente no se detecta almidón en las deposiciones, indicando que la energía correspondiente al almidón no digerido a nivel proximal está siendo rescatada en el colon a través de los procesos de fermentación por la microbiota y la generación de ácidos grasos volátiles. Es probable que la actividad inhibidora de amilasa se sature rápidamente en el lumen intestinal, debido al hecho que las enzimas pancreáticas como la amilasa estén secretadas en exceso. Como es de esperar, muchos estudios muestran una menor glicemia e insulinemia post-prandial, pero tales efectos se pueden conseguir incorporando un mayor contenido de fibra en el alimento o usando almidones con mayor contenido de amilosa.

\section{CONCLUSIÓN}

El nivel de evidencia científica que avala el uso de los tres productos evaluados para el control ponderal es escaso. Si bien el inhibidor de amilasa y el inhibidor de proteasa parecen funcionar in vitro, los ensayos clínicos realizados en sujetos normopeso u obesos no muestran ningún efecto sobre el control ponderal, aún si el inhibidor de amilasa parece impactar positivamente sobre el 
control de la glicemia e insulinemia. En caso del Olibra, los resultados son más discrepantes y más estudios son necesarios para confirmar su eventual efecto sobre el control del peso corporal.

\section{RESUMEN}

Paralelamente al rápido aumento de la prevalencia de sobrepeso y obesidad han surgido en el mercado múltiples productos que prometen estimular la pérdida de peso sin modificar la dieta y estilo de vida de los individuos. El objetivo de este trabajo es evaluar la evidencia científica que apoye el uso de tres productos disponibles en Chile para el control ponderal y la reducción de sobrepeso y obesidad: una emulsión lipídica que estimula el freno ileal, un inhibidor de proteasa y un inhibidor de amilasa. Los resultados indican que el inhibidor de amilasa y el inhibidor de proteasa, si bien parecen funcionar in vitro, no muestran ningún efecto sobre el control ponderal en los ensayos clínicos realizados tanto en sujetos normopeso como en obesos. El nivel de evidencia que apoya el uso de estos productos para el control ponderal es por lo tanto insuficiente. En caso de la emulsión lipídica, los resultados son conflictivos y más estudios son necesarios para confirmar su eventual efecto sobre el control del peso corporal.

Pañabras clave: suplementos nutricionales, sobrepeso, obesidad.

Dirigir la correspondencia a:

Profesor

Martin Gotteland, $\mathrm{PhD}$

Lab. de Microbiología y Probióticos

Instituto de Nutrición y Tecnología

de los Alimentos (INTA)

Universidad de Chile.

El Líbano 5524, Macúl, Santiago, Chile

F: 56-2-9781471

E-mail: mgottela@inta.cl
Agradecimientos: Financiado por Fondecyt 1080519.

BIBLIOGRAFÍA

1. Vio F, Albala C. Nutrition transition in Chile: a case study. In: FAO UN, Editor, Globalization of food systems in developing countries: impact on food security and nutrition. Rome: FAO Food and Nutrition Paper; 2004. p. 275-84.

2. World Health Organization WHO. Obesity and overweight. W.M. Centre, Editor. 2006, WHO.

3. Encuesta Nacional de Salud, Ministerio de Salud and Instituto Nacional de Estadísticas, Editors. 2003.
4. Blanck HM, Khan LK, Serdula MK. Use of nonprescription weight loss products: results from a multistate survey. JAMA 2001; 286: 930-5.

5. Maljaars PW, Peters HP, Mela DJ, Masclee AA. Ileal brake: a sensible food target for appetite control. A review. Physiol Behav 2008; 95: 271-81.

6. Murphy KG, Bloom SR. Gut hormones and the regulation of energy homeostasis. Nature 2006; 444: 854-9.

7. Carey MC, Small DM, Bliss CM. Lipid digestion and absorption. Annu Rev Physiol 1983; 45: 651-77.

8. Knutson L, Koenders D, Fridblom H, Vilberg A, Sein A, Lennernäs H. Gastrointestinal metabolism of a vegetable-oil emulsion in healthy subjects. Am J Clin Nutr 2010; 92: 515-24.

9. Burns AA, Livingstone MB, Welch RW, Dunne A, Robson PJ, Lindmark L, et al. Short-term effects of yoghurt containing a novel fat emulsion on energy and macronutrient intakes in non-obese subjects. Int J Obes Relat Metab Disord 2000; 24: 1419-25.

10. Burns, A.A., Livingstone MB, Welch RW, Dunne A, Reid CA, Rowland IR. The effects of yoghurt containing a novel fat emulsion on energy and macronutrient intakes in non-overweight, overweight and obese subjects. Int J Obes Relat Metab Disord 2001; 25: 1487-96.

11. Burns AA, Livingstone MB, Welch RW, Dunne A, Rowland IR. Dose-response effects of a novel fat emulsion (Olibra) on energy and macronutrient intakes up to $36 \mathrm{~h}$ post-consumption. Eur J Clin Nutr 2002; 56: 368-77.

12. Diepvens K, Steijns J, Zuurendonk P, WesterterpPlantenga MS. Short-term effects of a novel fat emulsion on appetite and food intake. Physiol Behav 2008; 95: 114-7.

13. Logan CM, McCaffrey TA, Wallace JM, Robson PJ, Welch RW, Dunne A, et al. Investigation of the medium-term effects of Olibra trade mark fat emulsion on food intake in non-obese subjects. Eur J Clin Nutr 2006; 60: 1081-91.

14. Diepvens K, Soenen S, Steijns J, Arnold M, Westerterp-Plantenga M. Long-term effects of consumption of a novel fat emulsion in relation to body-weight management. Int J Obes (Lond) 2007; 31: 942-9.

15. Haenni A, Sundberg B, Yazdanpandah N, Viberg A, Olsson J. Effect of fat emulsion (Fabuless) on orocecal transit time in healthy men. Scand J Gastroenterol 2009; 44: 1186-90.

16. Smit HJ, Keenan E, Kovacs EM, Wiseman SA, Peters HP, Mela DJ, et al. No efficacy of processed Fabuless (Olibra) in suppressing appetite or food 
intake. Eur J Clin Nutr 2011; 65: 81-86.

17. Szelényi Z. Cholecystokinin: role in thermoregulation and other aspects of energetics. Clin Chim Acta 2010; 411: 329-35.

18. Kissileff HR, Pi-Sunyer FX, Thornton J, Smith GP. C-terminal octapeptide of cholecystokinin decreases food intake in man. Am J Clin Nutr 1981; 34: 154-60.

19. Ahima RS, Antwi DA. Brain regulation of appetite and satiety. Endocrinol Metab Clin North Am 2008; 37: 811-23.

20. Delzenne N, Blundell J, Brouns F, Cunningham K, De Graaf K, Erkner A, et al. Gastrointestinal targets of appetite regulation in humans. Obes Rev 2010; 11: 234-50.

21. Gumbmann MR, Dugan GM, Spangler WL, Baker EC, Rackis JJ. Pancreatic response in rats and mice to trypsin inhibitors from soy and potato after shortand long-term dietary exposure. J Nutr 1989; 119: 1598-609.

22. Komarnytsky S, Cook A, Raskin I. Potato protease inhibitors inhibit food intake and increase circulating cholecystokinin levels by a trypsin-dependent mechanism. Int J Obes 2011; 35: 236-43.

23. Hill AJ, Peikin SR, Ryan CA, Blundell JE. Oral administration of proteinase inhibitor II from potatoes reduces energy intake in man. Physiol Behav 1990; 48: 241-6.

24. Schwartz JG, Guan D, Green GM, Phillips WT. Treatment with an oral proteinase inhibitor slows gastric emptying and acutely reduces glucose and insulin levels after a liquid meal in type II diabetic patients. Diabetes Care 1994; 17: 255-62.

25. Peters HP, Foltz M, Kovacs EM, Mela DJ, Schuring EA, Wiseman SA. The effect of protease inhibitors derived from potato formulated in a minidrink on appetite, food intake and plasma cholecystokinin levels in humans. Int J Obes 2011; 35: 244-50.

26. Merchant A, Vatanparast H, Barlas S, Dehghan M, Ali Shah S, De Koning L, et al. Carbohydrate intake and overweight and obesity among healthy adults. J Am Diet Assoc 2009; 109: 1165-72.

27. Wheeler ML, Pi-Sunyer FX. Carbohydrate issues: type and amount. J Am Diet Assoc 2008; 108 (Suppl 1): S34-9.

28. Naresh J, Boivin M, Zinsmeister A, Brown M, Malagelada J, Dimagno E. Effect of ileal perfusion of carbohydrates and amylase inhibitor on gastroin- testinal hormones and emptying. Gastroenterology 1989: 96: 377-87.

29. Granun PE. Studies on $\alpha$-amylase inhibitor in foods. Food Chem 1979; 4: 173-8.

30. Marshall J, Lauda C. Purification and properties of phaseolamina, an inhibitor of $\alpha$ - amylase, from kidney bean, Phaseolus vulgaris. J Biol Chem 1975; 250: 8030-37.

31. Layer P, Carlson G, Dimagno E. Partially purified white bean amylase inhibitor reduces starch digestion in vitro and inactivates intraduodenal amylase in humans. Gastroenterology 1985; 88: 1895-902.

32. Hollenbeck C, Coulston A, Quan R, Becker T, Vreman $\mathrm{H}$, Stevenson D et al. Effects of a commercial starch blocker preparation on carbohydrate digestion and absorption: in vivo and in vitro studies. Am J Clin Nutr 1983; 38: 498-503.

33. Layer P, Zinsmeister A, Dimagno E. Effects of decreasing intraluminal amylase activity on starch digestion and postprandial gastrointestinal function in humans. Gastroenterology 1986; 91: 41-8.

34. Boivin M, Flourie B, Rizza R, Vay Liang G, Dimagno E. Gastrointestinal and metabolic effects of amylase inhibition in diabetics. Gastroenterology 1988; 94: 387-94.

35. Vinson J, Al Kharrat H, Shuta, S. Investigation of an amylase inhibitor on human glucose absorption after starch consumption. Open Nutraceutical J 2009; 2: 88-91

36. Bo Linn G, Santa Ana C, Morawski S, Fordtran J. Starch Blocker: their effect on calorie absorption from high starch meal. New Engl J Med 1982; 307: 1413-16.

37. Boivin M, Zinsmeister A, Vay Liang G, Dimagno E. Effects of a purified amylase inhibitor on carbohydrate metabolism after a mixed meal in healthy humans. Mayo Clin Proc 1987; 62: 249-55.

38. Diaz E, Aguirre C, Gotteland M. Efecto de un inhibidor de $\alpha$-amilasa sobre la reducción de peso de mujeres obesas. Rev Chil Nutr 2004; 31: 306-17.

39. Thom E. A randomized, double-blind, placebocontrolled trial of a new weight-reducing agent of natural origin. J Int Med Res 2000; 28: 229-33.

40. Udani J, Hardy M, Madsen DC. Blocking Carbohydrate Absorption and Weight Loss: A Clinical Trial Using Phase $2^{\mathrm{TM}}$ Brand Proprietary Fractionated White Bean Extract. Alternative Med Rev 2004; 9: 63-9. 